\title{
Effect of damage rate on the kinetics of void nucleation and growth by phase field modeling for materials under irradiations
}

\author{
Xuejian Ding, Jiejiang Zhao, Hao Huang, Shurong Ding, Yongzhong Huo* \\ Department of Mechanics and Engineering Science, Fudan University, Shanghai 200433, China
}

\begin{abstract}
The void formation and growth in materials under irradiations is studied by a modified Cahn-Hilliard equation coupled with the explicit nucleation algorithm. Through the numerical simulations, the stages of incubation, nucleation, growth and coalescence of the irradiation induced voids are clearly observed with a faster kinetics for stronger damage rate. There seems to exist a critical damage rate $\dot{g}_{v}^{c}$ at which the kinetics speeds up significantly. For smaller damage rates, very few voids can be nucleated. But the nucleated voids can grow rather large with its average radius growing as $R_{v} \propto t^{1 / \mathrm{d}}$. For stronger irradiations, much more voids could be nucleated, but they cannot grow very large before coarsening. The growth follows a much faster kinetics as $R_{v} \propto t^{2 / d}$. The critical damage rate $\dot{g}_{v}^{c}$ should be determined by the competition of the rate of diffusion and the rate of vacancy production due to irradiations.
\end{abstract}

Keywords: modified Cahn-Hilliard equation; explicit nucleation algorithm; vacancy generation and accumulation; void formation and growth kinetics; irradiation effects

\section{Introduction}

Irradiation-induced microstructures such as voids and fission gas-filled bubbles in nuclear fuels and materials which may cause swelling are critically important to the performance of nuclear reactors [1-4]. The conventional method or theory mostly used to study void evolution and ordering in irradiated metal is rate theory [3-4]. Due to the mean field character, rate theory has some limitations for the study of void nucleation and growth. Phase filed method (PFM) has provided a powerful tool to describe the microstructure evolutions under irradiations. And it can be easily integrated into multi-physics and multi-scale numerical algorithms for simulation of irradiation effects of nuclear fuels and materials [5-6].

The first phase field simulation of void evolution for irradiated metal was proposed in 2005 by using the Cahn-Hilliard (C-H) equation for vacancy concentration [7]. Void nucleation and growth was observed for metals with a supersaturated vacancy concentration through spinodal decomposition. By introducing a vacancy generation term to modify the $\mathrm{C}-\mathrm{H}$ equation, so to mimic the damage rate of irradiation, the stages of incubation, nucleation, growth and coalescence of the irradiation induced voids were simulated successfully [8-9]. The PFM is further developed

*Corresponding author. Tel.: +86 02155664171

E-mail address: yzhuo@fudan.edu.cn (Y.Z. Huo). 
to include the effect of irradiation induced interstitials [10-11], temperature gradient [12-13] and grain boundaries [14-15]. Cahn-Hilliard and/or Allen-Cahn equations with some modifications have been applied [8-19]. The results seem promising and could be well comparable with the well-established rate-theory treatment of void evolution [20].

However, up to now, the void nucleation process in most phase field simulations is based on the Langevin noise approaches instead of the classical nucleation model which is used in the rate theory [1-2]. As pointed out in [16-17], the free-energy change in the PFM could be better calibrated against the classical nucleation model. Moreover, as suggested in [18], under the realistic irradiation environment, it should be very difficult for point-defect solutions to reach concentration high enough to become unstable causing spinodal decomposition to occur. Instead, they can become metastable at much lower mean concentrations, but with fluctuations large enough to overcome the activation barrier separating the phases. Hence, it seems that the detailed behavior of stochastic fluctuations plays a determinant role in the evolution of its microstructure via the classical nucleation theory. Therefore, it is desired to perform the phase field simulation for the void evolution by using the classical nucleation method.

In phase field modeling, the nucleation of new phase in phase field modeling is often realized by introducing the so called Langevin noise terms to mimic the effect of thermal fluctuations [21, 23]. Explicit nucleation algorithm is also proposed based on the classical nucleation theory of phase transformations in [22-23]. In this work, the explicit nucleation algorithm will be used for the void nucleation. The effect of the irradiation induced generation source term on the behavior of the solution of the modified C-H equation will be studied in some details. For simplicity, only the vacancy concentration will be chosen as the only field variable satisfying a modified $\mathrm{C}-\mathrm{H}$ equation with a vacancy generation source term $[8-9,14]$. Models with interstitials will be studied in future works.

In the next section, the computational model is summarized. The modified $\mathrm{C}-\mathrm{H}$ equation is presented in Sect. 2.1. The vacancy generation source term will be defined in Sect. 2.2 as an irradiation damage rate related random function. The explicit nucleation algorithm for void nucleation is discussed in Sect. 2.3. In Section 3, the process of the void nucleation, growth and coarsening is simulated for a range of irradiation damage rates. The kinetics is studied by considering the evolutions of the void fraction, the void number and the average radius of voids together with the vacancy concentrations in matrix, in voids and in total. The transition of kinetics for lower and higher damage rates is clearly visible from the very different exponent as given in Sect. 3.2. Both the nucleation and growth of voids are found to be different for different exponent as detailed in Sect. 3.3. The conclusions are given in Section 4.

\section{Computational model for void evolution in irradiated materials}

\section{under isothermal condition}

Displacement cascades are often formed under neutron collisions in reactor conditions with a vacancy rich core and an interstitial rich out-shell. Since the interstitials have much larger mobility than vacancies as reported by experiments and molecular dynamics simulations [5-6], void evolutions are primarily controlled by the behavior of the irradiation induced vacancies. Thus, for simplicity, we shall take the vacancy as the only variable here similar as in $[8-9,14]$. 


\subsection{Modified Cahn-Hilliard equation for vacancy concentration}

The vacancy concentration $c(\boldsymbol{x}, t)$ satisfies the following modified C-H equation,

$$
\frac{\partial c(\boldsymbol{x}, t)}{\partial t}=\nabla \cdot\left(M \frac{1}{N} \nabla \frac{\delta F}{\delta c}\right)+\dot{g}_{i r r}
$$

where $M$ characterizes the mobility of vacancies, $N$ is the number of lattice sites per unit volume of the material. $\delta F / \delta c$ is the variation of the total free energy. $\dot{g}_{i r r}$ is the source term introduced to describe the generation of vacancies due to irradiations.

The mobility of vacancies will be taken as a constant as in [8-9]. More sophistic formulae are proposed in [11-15] to account for effects of grain boundaries, temperature effects and so on.

The total free energy, including the chemical free energy $f(c(\boldsymbol{x}, t))$ and the gradient energy, can be described by the Ginzburg-Landau [36] type free energy

$$
F=N \int_{V}\left[f(c(\boldsymbol{x}, t))+\frac{\kappa_{c}}{2}(\nabla c(\boldsymbol{x}, t))^{2}\right] d V
$$

where $\kappa_{c}$ is called the gradient coefficient. $f(c(\boldsymbol{x}, t))$ is the local free energy density and should be a double-well function of the vacancy concentration $c$. Without losing generality, we consider the chemical free energy as [9-14]

$$
f=k_{B} T[c \ln c+(1-c) \ln (1-c)]+E_{v}^{f} c(1-c)
$$

where $E_{v}^{f}$ is the interface coefficient, $k_{B}$ is Boltzmann's constant and $T$ is the absolute temperature. The chemical free energy, $f$ in Eq. (3), is a double-well function of concentration $c$. We get the two local minima $c=c_{e q}^{m}$ for the matrix material and $c=c_{e q}^{v}$ for the voids as vacancy clusters. The height between maximum and minima is the value of the energy barrier, denoted by $\Delta f_{0}$.

\subsection{The vacancy generation rate due to irradiations}

Following [14], the rate of generation of vacancies due to irradiation will be taken as

$$
\dot{g}_{\text {irr }}(c(\boldsymbol{x}, t))=\left\{\begin{array}{rccc}
0 & \text { if } c>c_{0} & \text { or } & R_{1}>P_{\text {casc }} \\
R_{2} V_{G} & \text { if } c \leq c_{0} & \text { and } & R_{1} \leq P_{\text {casc }}
\end{array}\right.
$$

where $R_{1}$ and $R_{2}$ are two random numbers generated uniformly between 0 and $1, c_{0}$ is the critical concentration of cascades and areas with $c>c_{0}$ are defined as voids, $P_{\text {casc }}$ is the probability of cascades occurring within a unit volume per unit time and $V_{G}$ is the maximum increase in vacancy concentration due to a cascade event.

To analyze the effect of the vacancy production, we can integrate Eq. (1) over a material volume $V$ as,

$$
\frac{d}{d t} \int_{V} c(\boldsymbol{x}, t) d V=\int_{\partial V} M \frac{1}{N} \nabla \frac{\delta F}{\delta c} \cdot d \mathbf{S}+\int_{V} \dot{g}_{i r r} d V
$$

Consider periodic boundary conditions as often used for phase field simulations, there should be 
no net gains or losses of vacancies through the boundary. So the first term on the right hand side, the surface integration should vanish and we have the increase of the average total vacancy density $c_{t}(t):=\int_{V} c(\boldsymbol{x}, t) d V / V$ in the material as,

$$
\frac{d}{d t} c_{t}(t)=\frac{1}{V} \int_{V} \dot{g}_{i r r} d V=\frac{1}{V} \int_{c \leq c_{0}} \dot{g}_{i r r} d V=\frac{1}{2} V_{G} P_{\text {casc }}\left(1-X_{v}(t)\right)
$$

where $X_{v}$ is the void fraction, i.e. the volume fraction of regions with $c>c_{0}$. The probability of cascades, $P_{\text {casc }}$ and the maximum increase in vacancy concentration due to a cascade event, $V_{G}$ appear together to produce the same effect in the vacancy generation in the model. Thus, we may define the damage rate as $\dot{g}_{v}:=V_{G} P_{\text {casc }} / 2$ to represent the net generation rate of vacancies due to irradiations.

Integrating Eq. (6) further, we have the average vacancy concentration in the material at time $t$ as,

$$
c_{t}(t)=\int_{0}^{t} \dot{g}_{v}\left(1-X_{v}(\tau)\right) d \tau
$$

Thus, for materials without voids, a constant damage rate will result in a linearly increasing vacancy concentrations, $c_{t}(t)=\dot{g}_{v} t$. As soon as some voids have formed, the increase of vacancy in the material slows down, because there is no net generation of vacancies in the void regions according to Eq. (4). However, the total available vacancies are still increasing continuously. Two time scales are present in Eq. (1), the rate of vacancy production determined by the damage rate, $\dot{g}_{v}$ and the rate of diffusion determined by the vacancy mobility $M$. As will be shown by our simulations, it is the competition of these two time scales that will determine the kinetics of the irradiation induced void formation process.

\subsection{Explicit nucleation algorithm}

The explicit nucleation algorithm proposed in [22] and will be used here for the void nucleation. A brief description will be given below. According to the classical nucleation theory, the free energy change due to a spherical shaped nucleus of radius $R$ is

$$
\Delta F=-4 \pi R^{3} \Delta f / 3+4 \pi R^{2} \sigma
$$

where $\Delta f(c, T)=f_{v}-f_{m}$ is the energy difference between the void and the matrix, which is a function of matrix vacancy concentration and temperature. $\sigma$ is the surface energy per unit area. A critical nucleus corresponds to the maximum of the energy because any larger nucleus will have a smaller energy and spontaneously grow. The size and energy of the critical nucleus are then

$$
R^{c}(c, T)=\frac{2 \sigma}{\Delta f} \quad \Delta F^{c}(c, T)=\frac{16 \pi}{3} \frac{\sigma^{3}}{\Delta f^{2}}
$$

Thus, the steady state nucleation rate per unit volume is obtained as

$$
J(c, T)=J_{0} e^{-\Delta F^{c}(c, T) / k_{B} T}
$$

where $k_{B}$ is Boltzmann constant and $T$ is the temperature. $J_{0}$ is the nucleation coefficient.

Note that if the irradiation induced interstitials are also considered, the void nucleation rate can be affected rather strongly as in $[1,2]$. In this work, we limit ourselves to above simplified rate 
equation.

Considering that the length and time scale of nucleation is much smaller than that of the phase field method, we follow [22] to divide a phase field cell into sub-cells of the size similar to the critical nucleus. Besides, we divided a phase field time step into characteristic nucleation time steps. It is assumed that nucleation is so infrequent that the event of formation of two nuclei in one phase field cell can be neglected. The further assumption is made that the growth rate of the newly formed nuclei is sufficient that subsequent nucleation of a second precipitate at a later time within a single phase field cell can be neglected. Thus, the rate at which a sub-cell is transformed to the void phase is given by Eq. (10). Then, the probability of the transformation of a phase-field cell in a phase-field time step is

$$
P_{T}=1-(1-J \Delta v / \beta)^{\Delta \tau \beta \Delta V / \Delta v} \cong 1-e^{-J \Delta V \Delta \tau}
$$

where $\Delta v$ is the volume of a sub-cell and $\Delta V$ is the volume of a phase-field cell. $\beta$ is the frequency at which vacancies migrate to the surface of a critical void nucleus and $\Delta \tau$ is the time step in the phase-field simulation. To actuate the nucleation event, at each time step a random number, $P_{r}$, with uniform distribution between 0 and 1 is generated for each phase-field cell. A phase-field cell is considered to transform only if $P_{T}>P_{r}$. After a phase-field cell is determined to transform, a predefined profile will be assigned to the phase field variables at that position, which resembles that of an equilibrated nucleus. The actual size of the equilibrated nucleus should depends on the vacancy concentration and temperature, and we adopt the minimum value which ensure the growth of the newly nucleus in the calculation. The geometry of the nucleus is chosen to approximate a circle in a discrete lattice. A depletion layer about the nucleus is chosen to be about half as wide as its diameter. The composition is reduced in the depletion layer by exactly the amount required to give the additional concentration necessary for the void phase to form in the nucleus.

For the whole material volume, the expectation value of nucleation over the time period $\Delta \tau$ is

$$
E_{n}(t):=\int_{t}^{t+\Delta \tau} \int_{V} P_{T} d V d t
$$

When the above nucleation algorithm is applied for two dimensional phase field simulations, the nucleation rate Eq. (10) needs to be modified as [22]

$$
J(c, T)=J_{0} e^{-\pi \sigma^{2} / k_{B} T \Delta f(c, T)}
$$

with $J_{0}$ the 2-d nucleation coefficient. Besides, as in the Ref.[27], the driving force for nucleation $\Delta f$ at a concentration $c$ is the vertical distance between the two parallel tangential lines on the energy curve.

The algorithm of the method involves the following steps:

(I) Choose a location for a nucleation site in the matrix;

(II) Calculate a nucleation probability $P_{T}$ using Eq. (12) and (14)

(III)Generate a uniform random number $P_{r}$ between 0 and 1;

(IV) Add a equilibrated nucleus to the chosen location if $P_{T}$ is great than $P_{r}$

(V) The composition is reduced in a depletion layer around the nucleus. 


\subsection{Final dimensionless equations}

Using the phase field grid size $l_{0}$ and the phase field time step $\Delta \tau=l_{0}^{2} / M \Delta f_{0}$, Eq. (1) is reduced to,

$$
\frac{\partial c^{*}}{\partial t^{*}}=\nabla^{* 2}\left(\frac{\partial f^{*}}{\partial c^{*}}-\kappa_{c}^{*} \nabla^{* 2} c^{*}\right)+\dot{g}_{i r r}^{*}
$$

The dimensionless the expectation value of nucleation Eq. (12) and the nucleation rate Eq. (13) in 2 dimension are

$$
E_{n}\left(t^{*}\right):=\int_{t^{*}}^{t^{*}+1} \iint_{\Omega^{*}} P_{T} d x^{*} d y^{*} d t^{*} \text { and } J^{*}=J_{0}^{*} e^{-s^{*} / \Delta f^{*}}
$$

where

$$
\begin{gathered}
x^{*}=x / l_{0}, y^{*}=y / l_{0}, t^{*}=t / \Delta \tau, \Delta \tau=l_{0}^{2} / M \Delta f_{0} \\
c^{*}=\left(c-c_{e q}^{m}\right) / \Delta c_{e q}, \Delta c_{e q}=c_{e q}^{v}-c_{e q}^{m}, \dot{g}_{i r r}^{*}=\dot{g}_{i r r} \Delta \tau \\
\kappa_{c}^{*}=\kappa_{c} /\left(l_{0}^{2} \Delta f_{0}\right), s^{*}=\pi \sigma^{2} /\left(k_{B} T \Delta f_{0}\right), J_{0}^{*}=J_{0} l_{0}^{2} \Delta \tau
\end{gathered}
$$

\section{Results and discussions}

In the present work, all the simulations are carried out in two dimensions, although the formulas can be achieved for three dimensions. The semi-implicit Fourier spectral method is employed [24] and periodic boundary conditions are applied to the system. The dimensionless parameters for explicit nucleation algorithm and the gradient energy coefficient are chosen as [27] to ensure reasonable results. The dimensionless parameters, grid size and time step for simulation are listed in Table 1.

The vacancy generation rate is related to the neutron (electron) particle flux and their energy cross sections. And they can vary more than two magnitudes in radiation environments such as nuclear reactors [1-2]. Thus, we shall study the void formation kinetics under constant dimensionless damage rates $\dot{g}^{*}=\dot{g}_{v} \Delta \tau=V_{G} P_{\text {casc }} \Delta \tau / 2$ between $10^{-4}$ and $5 \times 10^{-3}$. The qualitative behavior of nucleation, growth and coarsening of voids is described firstly, followed by detailed analysis of the effect of the damage rate.

Since only the dimensionless quantities will present in this section, we shall drop the star sign for simplicity.

Table 1

Dimensionless parameters, grid size and time step for simulation

\begin{tabular}{cc}
\hline & Value \\
\hline Nucleation coefficient $J_{0}^{*}[27]$ & 1850 \\
Energy of the critical nucleus $s^{*}[27]$ & 2.98
\end{tabular}




$\begin{array}{cc}\text { Gradient energy coefficient } \kappa_{c}^{*} & 0.5 \\ \text { Initial vacancy concentration } c^{*} & 0\left(c=c_{e q}^{m}\right) \\ \text { Grid size } & 128 \times 128 \\ \text { Time step } & 0.0001\end{array}$

\subsection{Void formation and growth under constant irradiations}

Fig. 1 shows the void morphology evolutions obtained from the phase-field simulation for $\dot{g}=5 \times 10^{-4}$. The red areas with $c>c_{0}=0.5$ are considered as the voids while the blue regions with $c<0.2$ are the matrix. In between are the phase boundaries with $0.2<c<0.5$. The stages of incubation (Fig. 1(a)), nucleation (Fig. 1(b-d)), growth (Fig. 1(b-e)) and coalescence (Fig. 1(f)) of the irradiation induced voids can be clearly identified. Similar characteristics of the void evolution process can be observed for all the damage rates studied here and is also qualitatively similar to the results in [8-9, 14]. Fig. 2 (a) depicts the time evolutions of the voids number density, $n_{v}$, and the porosity (the area fraction of void regions), $X_{v}$. The average vacancy concentrations in matrix, $c_{m}$, in voids, $c_{v}$ and in total, $c_{t}$ are shown in Fig. 2(b).

In the incubation stage (Stage I: $0<t<t_{n}^{*}$ ), no voids can be observed (Fig. 1(a)). Thus, the total vacancy concentration $c_{t}$ equals the matrix concentration $c_{m}$ and they increase linearly according to Eq. (7) due to the continuously generation of vacancies by constant irradiation as indicated by the solid line in Fig. 2 (b). With time going, first nucleation event occurs at $t_{n}^{s}$ (Fig. 1 (b)) and it comes to the stage of nucleation and growth (Stage II: $t_{n}^{s} \leq \mathrm{t}<t_{c}^{s}$ ). Immediately, the average vacancy concentration in void $c_{v}$ reaches closely to 1 , while the total concentration $c_{t}$ deviates from a linear function and from the matrix concentration $c_{m}$ (Fig. 2(b)). The void number density $n_{v}$ increases firstly for $t>t_{n}^{s}$ (Fig. 1(c,d)) due to the continuously nucleation but reaches a maximum $n_{v}^{M}$ at time $t_{n}^{f}$ (Fig. 1(d)) when the nucleation finished. It is obvious from Fig. 1(b-d) that the nucleated voids had grown simultaneously during this concurrent nucleation and growth stage $t_{n}^{s} \leq \mathrm{t} \leq t_{n}^{f}$ (Stage IIA). It is interesting to observe from Fig. 2(b) that the average matrix vacancy $c_{m}$ keeps increasing firstly after $t_{n}^{s}$ and then decreases after attaining a maximum at $t_{m}^{M}$. The value $c_{m}\left(t_{n}^{t}\right)$ is smaller than $c_{m}\left(t_{n}^{s}\right)$ as will be detailed later. We also found that the maximum concentration of the matrix (around 0.09) is far below the critical vacancy concentration of spinodal decomposition (around 0.2113). And the total process turn out to be a first-order phase transition.

After $t_{n}^{f}$ comes the stage of pure growth (Stage IIB: $t_{n}^{f}<t<t_{c}^{s}$ ) (Fig. 1(d,e)), in which the void number $n_{v}$ remains unchanged with continuously increasing of the porosity $X_{v}$ (Fig. 2(a)). Coalescence started after $t_{c}^{s}$ (Fig. 1(f)) with decreasing $n_{v}$ and increasing $X_{v}$ (Fig. 2(a)).

It is obvious from Fig. 2(a) that the kinetics of the irradiation induced void evolution has very different characteristics in the three different stages. Its characteristics and in particularly the effect of the damage rate will be studied in some details in the next.

\subsection{Kinetics of void nucleation and growth under irradiation}

The irradiation induced void nucleation and growth occurs in Stage II $\left(t_{n}^{t} \leq t<t_{c}^{s}\right)$ which can be further divided into two substages, IIA - the concurrent nucleation and growth stage $\left(t_{n} \leq \mathrm{t} \leq t_{n}^{\prime}\right)$ and 
IIB - the pure growth stage $\left(t_{n}^{f}<t<t_{c}^{s}\right)$. As shown in Fig. 3 in the scaled time, $\tau_{1}:=\left(t-t_{n}^{s}\right) /\left(t_{n}^{f}-t_{n}^{s}\right)$ for all 11 simulated damage rates, the porosity $X_{v}$ is always increasing in the whole period. However, at Stage IIA $\left(0<\tau_{1} \leq 1\right)$, the void number density $n_{v}$ is not necessarily monotonically increasing. A drop of $n_{v}$ in Fig. 3(b) implies the resolution of nucleated voids, a kind of ripening, e.g. around $\tau_{1} \approx 0.55$ for $\dot{g}=3 \times 10^{-4}$. Moreover, the nucleation does not occur in a continuous manner except at rather strong irradiations as indicated by the many plateaus in Fig. 3(b). At Stage IIB with $\tau_{1}>1$, no further nucleation events will take place, $n_{v}$ remains a constant until $t=t_{c}^{s}$ while $X_{v}$ increases continuously.

It is obvious from Fig. 3(a) that the stronger the irradiation, the faster the porosity increases. To quantify the kinetics, we follow [9] to fit the porosity $X_{v}(t)$ in Stage IIA with the following analytical expression originated from the Johnson-Mehl-Avrami-Kolmogorov (JMAK) equation [25-26],

$$
X_{v}(t)=X_{v}^{0}\left(1-\exp \left[-k\left(t-t_{n}^{s}\right)^{n}\right]\right)
$$

where $n>0$ is the Avrami exponent. $k>0$ is the rate constant. $X_{v}^{0}$ is a porosity after Stage IIA.

As shown in Fig. 4(a), the plots of $\log \left(-\log \left(1-X_{v}(t) / X_{v}^{0}\right)\right)$ vs $\log (t)$ for all simulated damage rates do have relatively good linearity in Stage IIA. Fig. 4(b) depicts the fit of PFM with Eq. (17) for $\dot{g}=5$ and $7.5 \times 10^{-4}$. It is obvious that they agree quite well until a time $t_{\text {sim }}$ deeply in the pure growth stage (Stage IIB), i.e. $t_{n}^{f}<t_{\text {sim }}<t_{c}^{s}$ as indicated in Fig. 4(b).

Clearly, Eq. (17) cannot be fitted in the whole Stage IIB because the porosity does not reach a steady value but keeps increasing after $t_{\text {sim }}$. Thus, there is some arbitrariness in choosing the constant $X_{v}^{0}$ to fit with Eq. (17) in a time interval. Moreover, due to the randomness introduced by the nucleation Eq. (13) and irradiations Eq. (4), the nucleation and growth process differs slightly for each simulation. Thus, for every damage rate, $1 \times 10^{-4} \leq \dot{g} \leq 5 \times 10^{-3}$, the simulations are made three times. The fitted growth exponents $n$ are shown in Fig. 5. And it is clear that the mean values of $n$ increases with the damage rate $\dot{g}$ from a value slightly smaller than 2 for $\dot{g} \leq 5 \times$ $10^{-4}$ to a value close to 3 for $\dot{g} \geq 7.5 \times 10^{-4}$. It seems that there is a critical damage rate $\dot{g}_{c}$ (dimensionless) between $5 \times 10^{-4}$ and $7.5 \times 10^{-4}$, at which the void formation process changed its kinetics. The dashed line in Fig. 5 and subsequent figures is drawn at $\dot{g}_{c}=6 \times 10^{-4}$ just for illustration purpose.

This change of void nucleation and growth kinetics for different damage rates $\dot{g}$ can be also observed from the maximal voids number $n_{v}^{M}:=n_{v}\left(t_{n}^{f}\right)$ in Fig. 6(a), the porosity $X_{v}^{n}=X_{v}\left(t_{n}^{f}\right)$ and average void radius $R_{v}^{n}=R_{v}\left(t_{n}^{f}\right)$ at the end of Stage IIA in Fig. 6(b). Fig. 5(a) depicts that $n_{v}^{M}$ always increases with $\dot{g}$. However, both the value of $n_{v}^{M}$ and the slope of its increasing are relatively small at $\dot{g}<\dot{g}_{c}$ and become much larger for $\dot{g}>\dot{g}_{c}$. Thus, very few voids are generated for $\dot{g}<\dot{g}_{c}$ while larger numbers of voids can be obtained at bigger ones, $\dot{g}>\dot{g}_{c}$. However, as shown in Fig. 6(b), $R_{v}^{n}$ decreases with $\dot{g}$ with much flatter slopes at $\dot{g}>\dot{g}_{c}$, while $X_{v}^{n}$ remains more or less the same for $\dot{g}<\dot{g}_{c}$, but increases rather strongly for $\dot{g}>\dot{g}_{c}$, after a sharp increase near $\dot{g}_{c}$.

The above results have indicated clearly that the void formation kinetics have rather different characteristics for lower and larger damage rates. Namely, a small number of voids is nucleated for $\dot{g}<\dot{g}_{c}$ and they can grow to rather large sizes at the end of the nucleation and growth stage, while the total porosity remain still quite small. For larger damage rates, $\dot{g}>\dot{g}_{c}$, a large number of voids are nucleated with their sizes remained relatively small during the whole nucleation and 
growth stage. The porosity at the stage end increases strongly with the damage rate. The different behavior seems to indicate that both the nucleation and growth processes should be rather different at lower and stronger damage rates as will be discussed in the next.

Note that our fitted Avrami exponent $n$ are not all the same as in $[9,14]$ due to the different nucleation techniques employed. According to [26], the Avrami exponent $n$ depends on both nucleation rate $J$ and growth rate. Thus the nucleation algorithm and phase-field equation will influence the growth exponent at the same time.

\subsection{Characteristics of void nucleation under different irradiations}

Since the explicit nucleation algorithm is used here, the nucleation of voids is determined by the vacancy concentration in the matrix through the nucleation rate defined by Eq. (10). The evolution of the average matrix concentration $c_{m}$ is plotted in Fig. 7(a) for 4 different damage rates. Although they are qualitatively very similar, the effect of $\dot{g}$ is clearly visible. For larger $\dot{g}$, the linear slopes at the beginning of irradiation is bigger in accordance with Eq. (7) and all the characteristic times such as the void nucleation start and finish $t_{n}^{\text {sf }}$ and the maximal matrix concentration $t_{\mathrm{m}}^{\mathrm{M}}$ are all shorter. Moreover, the concentrations to start the nucleation $c_{m}\left(t_{n}^{s}\right)$ becomes slightly bigger for stronger damage rates while the concentrations at the end of nucleation $c_{m}\left(t_{n}^{f}\right)$ seems unchanged and are always much smaller than $c_{m}\left(t_{n}^{s}\right)$. The maximal matrix concentration $c_{m}\left(t_{\mathrm{m}}^{\mathrm{M}}\right)$ is evidently bigger for larger damage rates. Thus, Fig. 7(a) shows clearly that for stronger damage rates, the matrix concentration has a bigger value during the whole concurrent nucleation and growth stage $t_{n}^{s} \leq \mathrm{t} \leq t_{n}^{f}$ (Stage IIA). Hence by Eq. (10), this should result in a larger nucleation rate.

It is very interesting to observe from Fig. 7(b) that the concentration difference, $\Delta c_{m}=c_{m}\left(t_{\mathrm{m}}^{\mathrm{M}}\right)-c_{m}\left(t_{n}^{\mathrm{s}}\right)$ is rather small for $\dot{g}<\dot{g}_{c}$ and jumps to a larger value for $\dot{g}>\dot{g}_{c}$ at the critical damage rate $\dot{g}_{c}$. Thus, we would expect a change of nucleation behavior around $\dot{g}_{c}$. As shown in Fig. 8, the expectation of nucleation of whole material area $E_{\mathrm{n}}$ of Eq. (15.1) increases with time near $t_{n}^{s}$ firstly and reaches a maximum $E_{n}^{M}$ around $t_{\mathrm{m}}^{\mathrm{M}}$ before they decrease to zero after $t_{n}^{f}$. Bigger damage rates will lead to larger expectations. It seems that the critical damage rate $\dot{g}_{c}$ should correspond to a unit maximum expectation value, i.e.

$$
E_{n}^{M}\left(\dot{g}_{c}\right)=1
$$

Thus, at lower damage rate $\dot{g}<\dot{g}_{c}$, the expectation value of nucleation is always smaller than one, i.e. $E_{\mathrm{n}}(t) \leq E_{n}^{M}<1$. Consequently, the void nucleation will take place discontinuously as observable from the plateaus in Fig. 3(b) and rather smaller number of voids can be nucleated as indicated in Fig. 6(a). The reason is certainly due to the very low vacancy concentration in matrix as shown in Fig. 7. For larger damage rates, $\dot{g}>\dot{g}_{c}, E_{n}^{n}>1$. Thus, there is always a time interval in which the expectation of nucleation is bigger than 1 due to the relatively larger matrix concentration. Thus, the nucleation can proceed more continuously and much more voids can form.

\subsection{Kinetics of growth: the average void radius}

The difference of the growth kinetics for lower and larger damage rates can be observed clearly 
from the evolution of the average void radius, $R_{v}(t)$ as well. As shown in Fig. 9(a) in the scaled time $\tau_{2}:=\left(t-t_{n}^{s}\right) /\left(t_{\text {sim }}-t_{n}^{s}\right)$, the radius of the initially nucleated voids at $t=t_{n}^{s}$ differs just very slightly for different damage rates. However, $R_{v}$ increases rather strongly for $\dot{g}<\dot{g}_{c}$ but changes very little for $\dot{g}>\dot{g}_{c}$ until the end of nucleation $t=t_{n}^{t}$. Moreover, the increases of $R_{v}$ seem to follow different laws in the pure growth stage $t>t_{n}^{f}$. Fig. 9(b) depicts that the void growth could be then relatively well fitted by

$$
R_{v}(t)=R_{v}\left(t_{n}^{f}\right)+K\left(t-t_{n}^{f}\right)^{(n-1) / d}
$$

Where $n$ is the corresponding Avrami exponent obtained by Eq. (17) and is shown in Fig. 5. $K$ is a positive constant and $d=2$ is the dimension of the phase field simulation. Thus, the average radius increases with $\left(t-t_{n}^{f}\right)^{1 / 2}$ at lower damage rates $\dot{g}<\dot{g}_{c}$, but changes to a linearly increasing function at $\dot{g}>\dot{g}_{c}$. Thus, in addition to the change of the characteristics of the void nucleation process, the void growth also changes its kinetics rather strongly around the critical damage rate $\dot{g}_{c}$.

\subsection{Discussion}

To convert to real units, the length scale $l_{0}$, time scale $\Delta \tau$, the height of the energy barrier $\Delta f_{0}$ and mobility $M=D / k_{B} T=D_{0} \exp \left(-E_{v}^{m} / k_{B} T\right) / k_{B} T$ must be given. As in ref. [10], we take copper as the example, the defect formation energy is set as $E_{v}^{f}=1 \mathrm{eV}$, the vacancy migration energy is $E_{v}^{m}=1.06 \mathrm{eV}$ and the vacancy diffusivity prefactor is $D_{v}^{0}=9.1 \times$ $10^{-16} \mathrm{~m}^{2} / \mathrm{s}$. If we take the length scale as $l_{0}=10 \mathrm{~nm}$, at $500 \mathrm{~K}$, the height of the energy barrier $\Delta f_{0}=0.22 \mathrm{eV}$, the diffusivity is $D=1.96 \times 10^{-16} \mathrm{~m}^{2} / \mathrm{s}$ and the time scale $\Delta \tau=l_{0} / M \Delta f_{0}=$ $0.1 \mathrm{~s}$. Assuming these values, the damage rates $\dot{g}_{v}=\dot{g} / \Delta \tau$ are between $10^{-3}$ and $5 \times 10^{-2} \mathrm{dpa} / \mathrm{s}$. These Frenkel pair production rates are comparable to electron ion beam displacement rates of $\sim 10^{-3} \mathrm{dpa} / \mathrm{s}$ [28]. The interfacial energy of the void free surface $\sigma=\sqrt{k_{B} T \Delta f_{0} s^{*} / \pi}=$ $0.44 \mathrm{ev} / \mathrm{nm}^{2}$.

Based on the above parameters, we can estimate the characteristic times for void nucleation. For example, under damage rate $\dot{g}_{v}=5 \times 10^{-3} \mathrm{dpa} / \mathrm{s}$, the time to start void nucleation is $t_{\mathrm{ns}}=1430 \mathrm{~s}$ and the time to end is $t_{\mathrm{nf}}=2300 \mathrm{~s}$. Besides, the average radius of the voids grow to $83 \mathrm{~nm}$ at $t=3170 \mathrm{~s}$ $\left(\tau_{1}=2\right)$. With the increase of the damage rate, the average radius of voids vary from $128 \mathrm{~nm}$ to $48 \mathrm{~nm}$ at $\tau_{1}=2$. These values seem comparable with experimental observations [29]. If we consider that our sample has a thickness, such as $z=1 \mathrm{~nm}$, the maximum void densities is 2.44 40.3× $10^{15} / \mathrm{cm}^{3}$, which is also comparable to the experiment data $\sim 10^{15} / \mathrm{cm}^{3}$ [29]. Moreover, the observation that lager void density is obtained for larger damage rate is also similar to the experiment data[29].

For the dimensionless system Eq. $(14,15)$, the nucleation kinetics is affected strongly by the values of the dimensionless nucleation coefficient $J_{0}^{*}$ and the dimensionless energy of the critical nucleus $s^{*}$. A larger $J_{0}^{*}$ and smaller $s^{*}$ will result in a stronger nucleation rate by Eq. (15.2) and a bigger expectation value $E_{n}(t)$ by Eq. (15.1). Thus, we would have a smaller dimensionless damage rate $\dot{g}_{c}$ from $E_{n}\left(\dot{g}_{c}\right)=1$ than the value between $5 \times 10^{-4}$ and $7.5 \times 10^{-4}$ as predicted by the present simulation. The dimensionless gradient energy coefficient $\kappa_{c}^{*}$ affects more on the void-matrix interfacial thickness and should have little effects on $\dot{g}_{c}$. The critical damage rate $\dot{g}_{c}$ is between $5 \times 10^{-3}$ and $7.5 \times 10^{-3} \mathrm{dpa} / \mathrm{s}$. 
However, the above result is only based on simulations with a rather simplified phase field model using the explicit nucleation algorithm. The formulation of the void, as a first order transition, could be simulate by the coupling models. And our results could fit the experiment data qualitative and quantitative to some degree. Many important effects are not considered yet $[1,2]$, e.g., the interstitials, temperature, network dislocations, etc. The gradient coefficient and the surface tension used in our simulation are directly from the literature [27] and are not good enough for voids considered here [16]. Besides, the material under simulation is taken to be homogeneous, isotropic and stress free. So the effects of grain boundaries, crystal structure and mechanical constraints are not considered either. Therefore, much more works should be done to clarify the observed critical damage rates and the related changing of kinetics.

\section{Conclusions}

The explicit nucleation algorithm based on the classical nucleation theory has been successfully utilized with a modified Cahn-Hilliard equation to simulate the accumulation process of the irradiation induced vacancies in materials under irradiations. The results show clearly that the accumulation process consists of stages of incubation, nucleation, growth and coalescence. Faster kinetics of the accumulation process is observed for stronger irradiation damage rates. The kinetics is mainly determined by the competition of the rate of diffusion and the rate of vacancy production due to irradiations.

Moreover, the simulations seem to indicate that there exist a critical damage rate for vacancy production $\dot{g}_{v}^{c}$ at which the kinetics speeds up significantly. Namely, at damage rates lower than $\dot{g}_{v}^{c}$, the rate of void nucleation is rather small, so very few voids can be nucleated. But the nucleated voids can grow rather large with their average radius $R_{v} \propto t^{1 / \mathrm{d}}$. For irradiations bigger than $\dot{g}_{v}^{c}$, the rate of void nucleation becomes rather large with the maximal expectation of nucleation in the sample be greater than one. Hence, much more voids could be nucleated, but they cannot grow very large before coarsening. The growth follows a much faster kinetics with $R_{v} \propto t^{2 / d}$.

\section{Acknowledgement}

This work is supported by the National Science Foundation of China (No. 11272092, $11461161008,11572091)$. The authors also gratefully acknowledge support from Dr. Shenyang $\mathrm{Hu}$ (PNNL) for providing us his codes and useful discussions. Special thanks should go to Prof. C.H. Woo and Dr. A.A. Semenov (City U HK) for valuable discussions and suggestions.

\section{References}

[1] Olander D R. Fundamental aspects of nuclear reactor fuel elements[R]. California Univ., Berkeley (USA). Dept. of Nuclear Engineering, 1976.

[2] Was G S. Fundamentals of Radiation Materials Science[M]// Springer Berlin Heidelberg, 
2007:52.

[3] Brailsford A D, Bullough R. The rate theory of swelling due to void growth in irradiated metals[J]. Journal of Nuclear Materials, 1972, 44(72):121-135.

[4] Bullough R, Nelson R S, Bullough R, et al. Voids in irradiated metals[J]. Physics in Technology, 1974, 5(1):29-67.

[5] Li D, Li Y, Hu S, et al. Predicting Thermal Conductivity Evolution of Polycrystalline Materials Under Irradiation Using Multiscale Approach[J]. Metallurgical \& Materials Transactions A Physical Metallurgy \& Materials Science, 2012, 43(3):1060-1069.

[6] Tonks M R, Gaston D, Millett P C, et al. An object-oriented finite element framework for multiphysics phase field simulations[J]. Computational Materials Science, 2012, 51(1):20-29.

[7] Yu H C, Lu W. Dynamics of the self-assembly of nanovoids and nanobubbles in solids[J]. Acta Materialia, 2005, 53(6): 1799-1807.

[8] Hu S Y, Jr C H H, Hu S Y. Phase-field modeling of void lattice formation under irradiation[J]. Journal of Nuclear Materials, 2009, 394(2):155-159.

[9] Rokkam S, El-Azab A, Millett P, et al. Phase field modeling of void nucleation and growth in irradiated metals[J]. Modelling \& Simulation in Materials Science \& Engineering, 2009, 17.

[10] Millett P C, El-Azab A, Rokkam S, et al. Phase-field simulation of irradiated metals Part 1: Void kinetics.[J]. Comput.mater.sci, 2011, 50.

[11]LI, YuLan, HU, et al. Phase-field modeling of void evolution and swelling in materials under irradiation[J]. Science China(Physics, 2011, 54(5):856-865.

[12]Hu S Y, Henager C H. Phase-field simulation of void migration in a temperature gradient[J]. Acta Materialia, 2010, 58(9):3230-3237.

[13] Li, Yulan, $\mathrm{Hu}$, et al. Phase-field Modeling of Void Migration and Growth Kinetics in Materials under Irradiation and Temperature Field.[J]. Journal of Nuclear Materials, 407(2):119-125, 2010, 407(2):119-125.

[14] Millett P C, Rokkam S, El-Azab A, et al. Void nucleation and growth in irradiated polycrystalline metals: a phase-field model[J]. Modelling \& Simulation in Materials Science \& Engineering, 2009, 17(6):064003.

[15] Li D, Li Y, Hu S, et al. Predicting Thermal Conductivity Evolution of Polycrystalline Materials Under Irradiation Using Multiscale Approach[J]. Metallurgical \& Materials Transactions A Physical Metallurgy \& Materials Science, 2012, 43(3):1060-1069.

[16] Semenov A A, Woo C H. Interfacial energy in phase-field emulation of void nucleation and growth[J]. Journal of Nuclear Materials, 2011, 411(1): 144-149.

[17] Semenov A A, Woo C H. Phase-field modeling of void formation and growth under irradiation[J]. Acta Materialia, 2012, 60(17):6112-6119.

[18] Semenov A A, Woo C H. Modeling void development in irradiated metals in the phase-field framework[J]. Journal of Nuclear Materials, 2014, 454(1): 60-68.

[19]El-Azab A, Ahmed K, Rokkam S, et al. Diffuse interface modeling of void growth in irradiated materials. Mathematical, thermodynamic and atomistic perspectives[J]. Current Opinion in Solid State \& Materials Science, 2014, 18(2):90-98.

[20]Xiao Z H, Semenov A A, Woo C H, et al. Single void dynamics in phase field modeling[J]. Journal of Nuclear Materials, 2013, 439(1): 25-32.

[21]Lifshitz E M, Pitaevskii L P. Statistical Physics, 3rd edn, part 1[J]. Landau and Lifshitz Course of Theoretical Physics, 1980, 5. 
[22] Simmons J P, Shen C, Wang Y. Phase field modeling of simultaneous nucleation and growth by explicitly incorporating nucleation events[J]. Scripta Materialia, 2000, 43(10):935-942.

[23] Heo T W, Chen L Q. Phase-Field Modeling of Nucleation in Solid-State Phase Transformations[J]. JOM: The Journal of The Minerals, Metals \& Materials Society (TM, 2014, 66(8):1520-1528.

[24]Chen L Q, Shen J. Applications of semi-implicit Fourier-spectral method to phase field equations[J]. Computer Physics Communications, 1998, 108(2):147-158.

[25]Porter D A, Easterling K E, Sherif M. Phase Transformations in Metals and Alloys, (Revised Reprint)[M]. CRC press, 2009.

[26]Christian J W. The theory of transformations in metals and alloys[M]. Newnes, 2002.

[27] Shen C. The fundamentals and applications of phase field method in quantitative microstructural modeling[D]. The Ohio State University, 2004.

[28]Benfu HU, Heishichiro T, Hisatoshi I. Behavior of void swelling and radiation-induced segregation in fe-cr-mn alloy system under electron irradiation. Acta Metallurgica Sinica [J]. Acta Metallurgica Sinica, 1990, 20(1):60-74.

[29]Hudson J A, Mazey D J, Nelson R S. Void formation in nickel during $20 \mathrm{MeV} \mathrm{C++}$ irradiation at $525{ }^{\circ} \mathrm{C}[\mathrm{J}]$. Journal of Nuclear Materials, 1971, 41(3):241-256. 


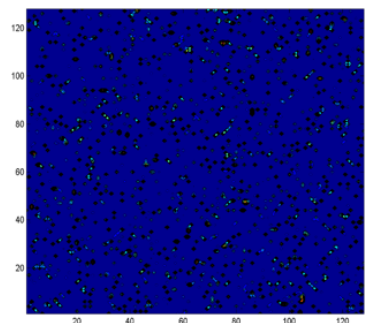

(a) $t=1$

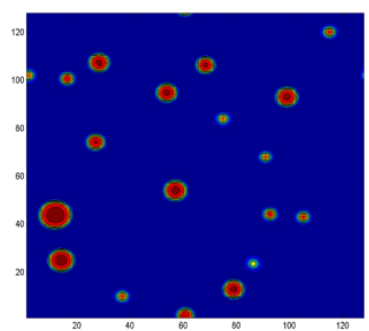

(d) $t=231$

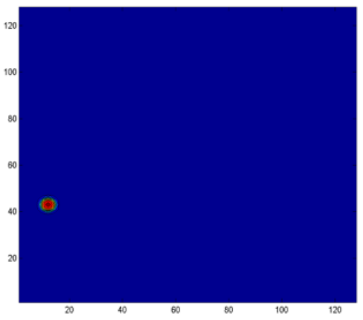

(b) $t=159$

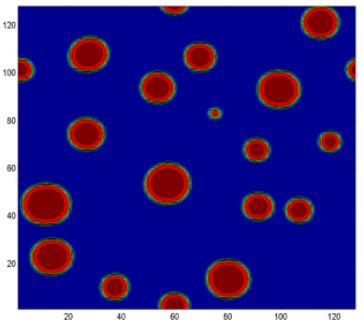

(e) $t=361$

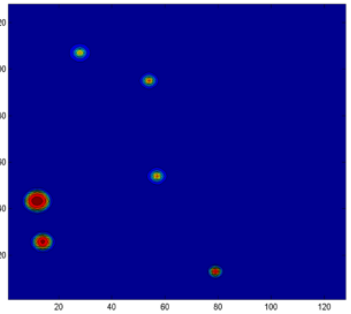

(c) $t=188$

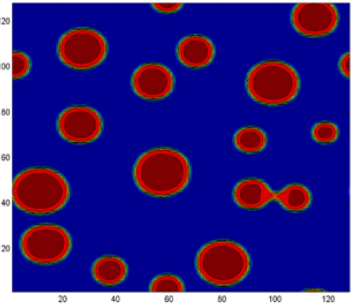

(f) $t=507$

Fig. 1. The void morphology evolutions for material under constant damage rate $\dot{g}=5 \times 10^{-4}$. The nucleation started at $t_{n}^{t}=149$ and finished at $t_{n}^{t}=230$. The coalescence of voids started at $t_{c}^{t}=430$. The red areas with $c>c_{0}=0.5$ are considered as the voids while the blue regions with $c<0.2$ are the matrix.
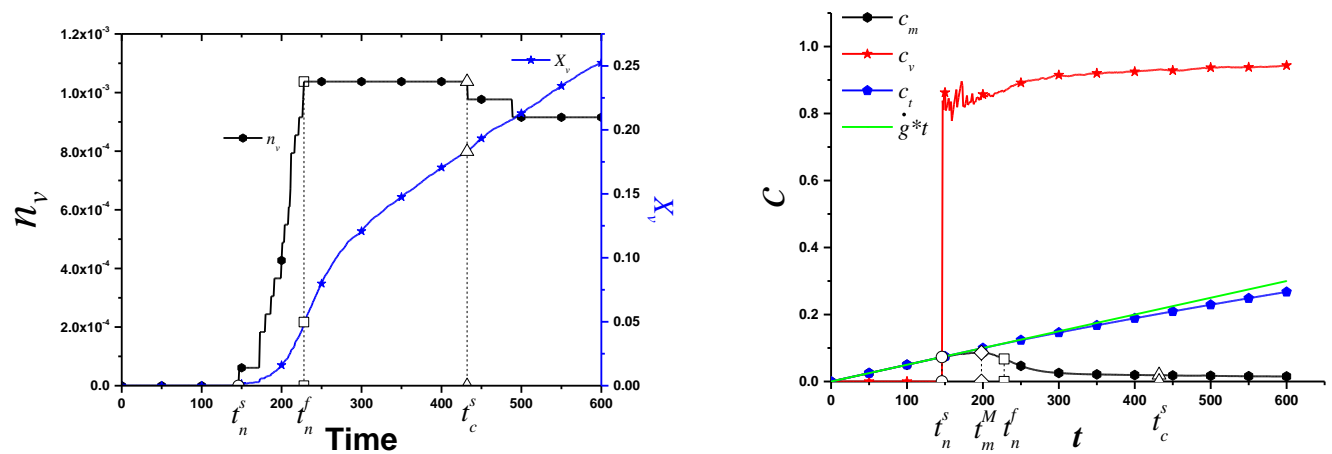

Fig. 2. The time evolutions of (a) the voids number density, $n_{v}$, the void fraction, $X_{v}$, (b) the average vacancy concentrations in matrix, $c_{m}$, in void, $c_{v}$ and in total, $\bar{c}$ for $\dot{g}=5 \times 10^{-4}$ together with the linearly increasing vacancy concentration. The characteristic times $t_{n}^{s}, t_{n}^{M}, t_{n}^{f}$ and $t$ ${ }_{c}^{s}$ are shown by circles, diamonds, squares and triangles respectively.
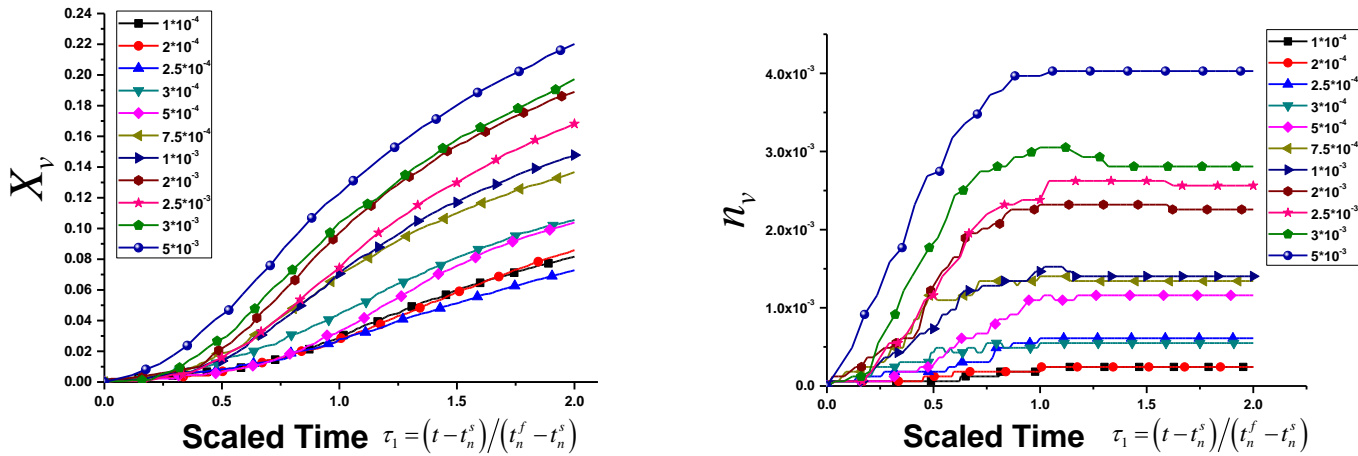

Fig. 3. The evolutions of (a) the void fractions $X_{v}$, (b) the void number densities $n_{v}$ with respect the 
scaled time $\tau_{1}:=\left(t-t_{n}^{s}\right) /\left(t_{n}^{f}-t_{n}^{s}\right)$ for eleven damage rates.
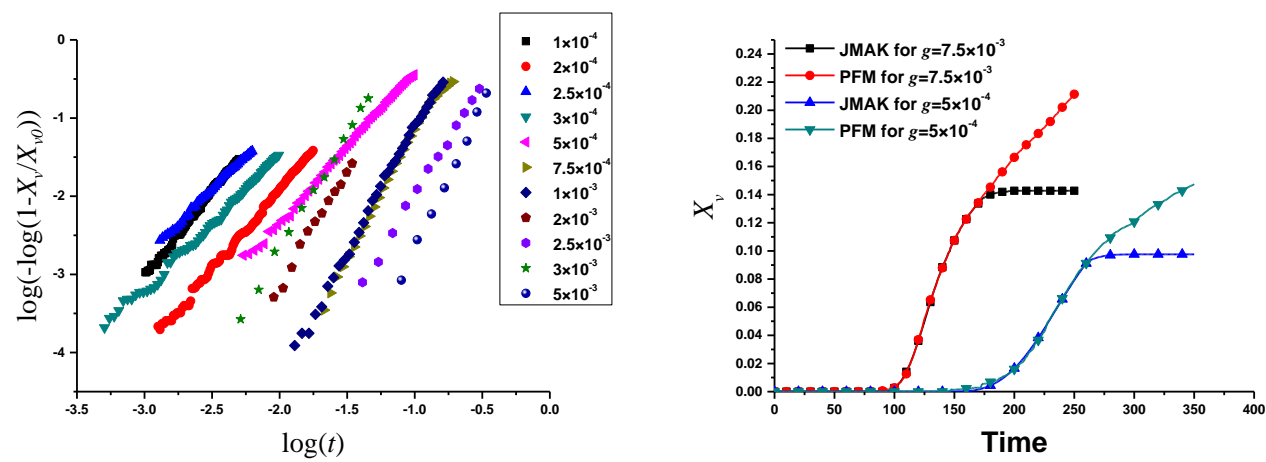

Fig. 4. (a) Log-log plot of phase field kinetics under different damage rates. (b) Fit results with damage rates $\dot{g}=5$ and $7.5 \times 10^{-4}$ compared with phase field results. The fitting is good for $t_{n}^{s}$ $<t<t_{\text {sim }}$. For $\dot{g}=7.5 \times 10^{-4}, t_{n}^{t}=90, t_{\text {sim }}=155, \mathrm{X}_{\mathrm{v}}^{0}=0.14277, k=3.056, n=2.886$; for $\dot{g}=5 \times 10^{-4}$, $t_{n}^{s}=149, t_{\text {sim }}=255, X_{v}^{0}=0.09756, k=2.348, n=2.021$;

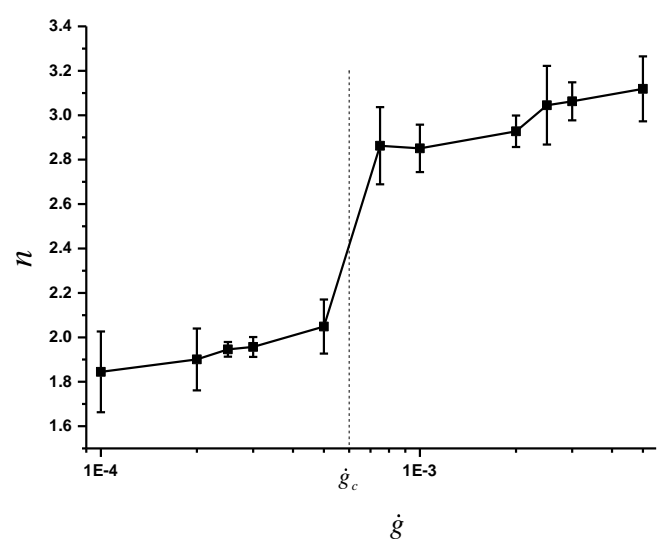

Fig. 5. The exponent $\mathrm{n}$ under different damage rates. Three simulations are performed for each damage rate and their mean value and error bar are shown. The solid line linking the mean values and the dashed line indicating the critical damage rate drawn at $\dot{g}_{c}=6 \times 10^{-4}$ are just guide for the eyes.
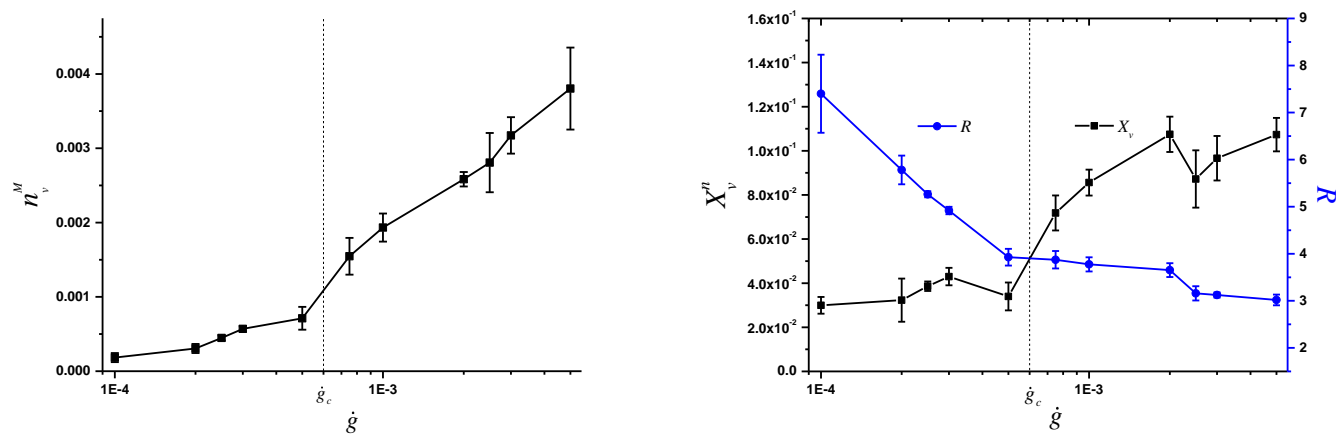

Fig. 6. Damage rate dependence of (a) the maximal void number density $n_{v}^{M}:=n_{v}\left(t_{n}^{f}\right)$ (b) the void fraction $X_{v}^{n}=X_{v}\left(t_{n}^{f}\right)$ and the average void radius $R_{v}^{n}=R_{v}\left(t_{n}^{f}\right)$ at the end of nucleation. 

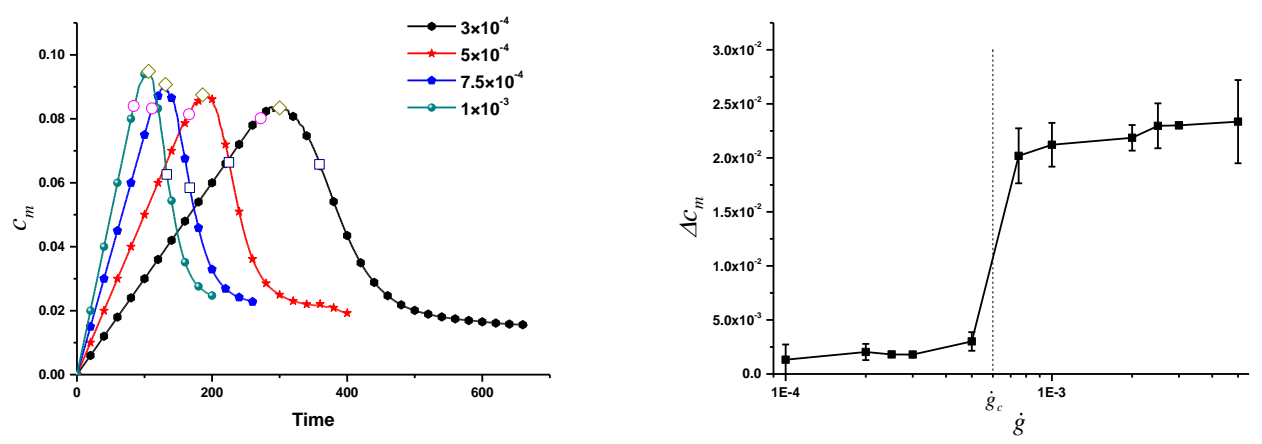

Fig. 7. (a) The evolution of the average matrix concentration for 4 different damage rates. The characteristic times $t_{n}^{s f}$ and $t_{m}^{M}$ are shown by circles, diamonds and squares, respectively. (b) The matrix concentration difference $\Delta c_{m}=c_{m}\left(t_{\mathrm{m}}^{\mathrm{M}}\right)-c_{m}\left(t_{n}^{\mathrm{s}}\right)$ under different damage rates. The solid and dashed lines are guides for the eyes.

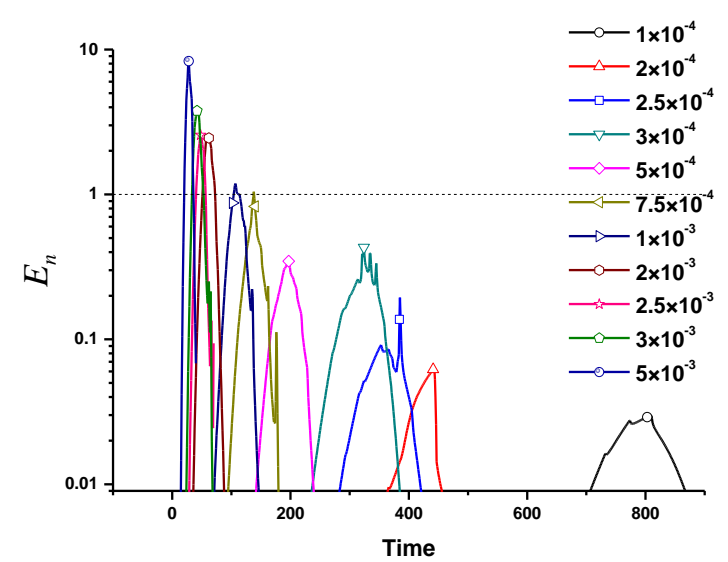

Fig. 8. The evolution of the expectation of nucleation under different damage rates.
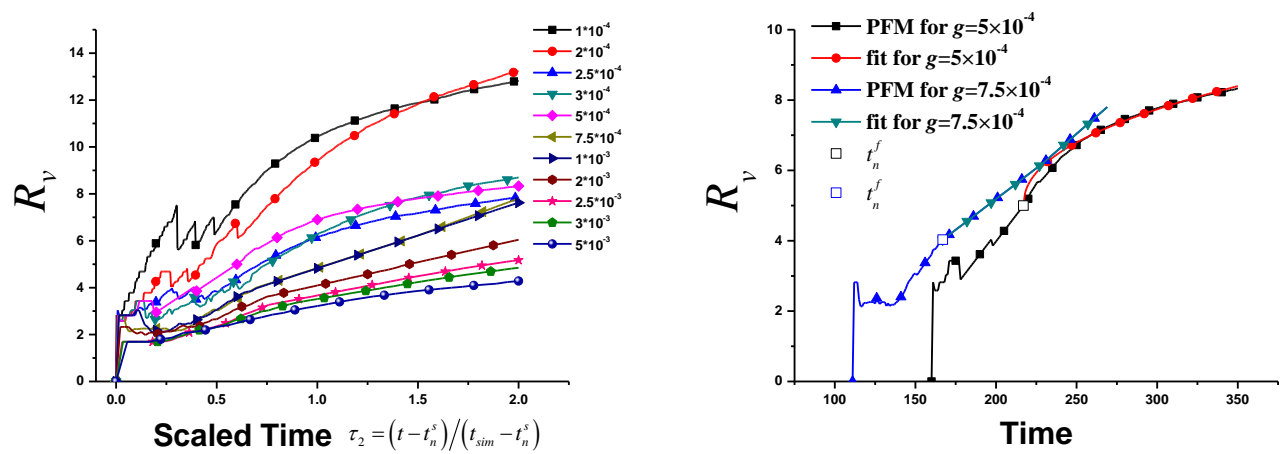

Fig. 9. The evolution of the average void radius (a) for 11 damage rates with respect to the scale time $\tau_{2}:=\left(t-t_{n}^{s}\right) /\left(t_{\text {sim }}-t_{n}^{s}\right)$, (b) for $\dot{g}=5$ and $7.5 \times 10^{-4}$ compared to fitting by Eq. (17) with $n=2$ and 3 , respectively. 